\title{
Présentation d'une carte de soins axée sur le patient pour la chirurgie colorectale : étude pilote qualitative des expériences des patients et des infirmières en oncologie chirurgicale
}

\author{
Jason Sawyer, Frances C. Wright, Shari L. Moura, \\ Barbara-Anne Maier et Margaret I. Fitch \\ Abrégé
}

Les cartes de soins existent depuis de nombreuses années. Des intervenants clés de notre établissement ont conçu et mis en œuvre une carte de soins pour les patients subissant une chirurgie pour un cancer colorectal. Cette étude pilote de nature qualitative et descriptive avait deux buts. Premièrement, celui de comprendre, au moyen de journaux de bord et d'entrevues, l'expérience vécue des patients soignés en vertu de la carte de soins nouvellement mise en ouvre. Deuxièmement, celui de décrire, au moyen d'un groupe de discussion, les expériences des infirmières en oncologie chirurgicale prodiguant les soins à ces patients. Les résultats de notre étude pilote indiquaient que les patients appréciaient le fait de disposer d'un document présentant les activités et les objectifs quotidiens et qu'ils avaient hâte de retourner chez eux, mais qu'ils étaient déçus du processus de planification des congés. De manière générale, les infirmières entretenaient des sentiments positifs vis-à-vis de la carte de soins, mais elles estimaient qu'elles auraient pu contribuer davantage aux étapes de sa conception et de sa planification. Dans l'ensemble, la mise en cuvre de la carte de soins axée sur le patient a été une réussite.

Les systèmes de soins de santé subventionnés par l'État, tel que celui du Canada, doivent composer avec des pressions croissantes en vue de maitriser la hausse des coûts associés à la fourniture des soins aux personnes qui en ont besoin et à fournir ces soins en temps opportun. Depuis le début du nouveau millénaire, l'un des secteurs d'intervention privilégiés de l'Ontario a été la réduction des temps d'attente en chirurgie pour diverses populations, y compris celle des patients atteints de cancer. Les écrits récents concernant la population de chirurgie colorectale rapportent qu'il est possible d'obtenir un séjour moyen de 2 jours (Basse et coll., 2000; Basse et coll., 2002). En 2004, le Ministère de la Santé et des Soins de longue durée (MSSLD) de l'Ontario a lancé un appel de propositions dans le cadre de sa Stratégie de réduction des temps d'attente pour trouver des organismes avançant des façons nouvelles et/ou novatrices de réduire les temps d'attente en chirurgie. Le Centre des sciences de la santé Sunnybrook a déposé une demande de subvention dans le cadre d'une proposition visant à réduire le séjour moyen pour la population d'oncologie chirurgicale colorectale. Son projet avait un double objectif : 1) instituer la pratique fondée sur des données probantes comme norme locale standard de prestation de soins aux patients subissant une chirurgie pour cancer colorectal; 2) saisir l'expérience vécue des patients et des infirmières en oncologie qui leur prodiguent des soins. Cet article est une présentation

\footnotetext{
Auteur pour la correspondance : Jason Sawyer, IPSA, Acute Pain Service, Centre des sciences de la santé Sunnybrook, 2075 Bayview Avenue, Toronto, Ontario, Canada M4N 3 M5 Téléphone: 416-480-6100 poste 2945, télécopieur: 416-480-6039, courriel : Jason.sawyer@sunnybrook.ca
}

Frances $C$. Wright, MD, MEd, FRCSC

Shari L. Moura, inf., M.Sc.inf., $C O N(C), C H P C N(C)$

Barbara-Anne Maier, inf., B.Sc.inf., $C O N(C)$

Margaret I. Fitch, inf., PhD des expériences initiales des patients et des infirmières après l'introduction d'une carte de soins privilégiant l'amélioration de l'information destinée aux patients et/ou à la famille, la définition des buts des soins et la réduction du séjour moyen à la suite d'une chirurgie pour cancer colorectal.

\section{Contexte et recension des écrits}

\section{Élaboration de Jalons}

En juillet 2002, un groupe clé d'intervenants professionnels (chirurgiens-oncologues, infirmières en oncologie, infirmières en pratique avancée et professionnels de disciplines connexes) se sont réunis afin d'analyser et d'explorer les besoins en matière de soins, la norme de soins et la durée prévue du séjour (le séjour moyen) des patients subissant une chirurgie pour un cancer colorectal dans un centre de soins tertiaires. Ce groupe interprofessionnel s'était doté d'une double mission : (1) élaborer des stratégies adéquates en vue de faciliter la circulation, vers les patients et les membres de la famille, de l'information concernant le séjour moyen; (2) s'assurer que les professionnels de la santé participant aux soins postopératoires appliquent et renforcent cette information.

Pour l'accomplissement de la première facette de cette mission, nous avons effectué une recension des écrits, une analyse du contexte afin d'évaluer la pratique actuelle et une revue des lignes directrices sur les pratiques exemplaires d'autres établissements de chirurgie et enfin, nous avons défini la durée de séjour anticipée.

Notre recension des écrits portait sur les sujets suivants : séjour et chirurgie colorectale, modalités de contrôle de la douleur postopératoire, nutrition orale de stade précoce, mobilisation active des patients et besoins éducationnels des patients. Nous avons déterminé qu'en combinant l'analgésie épidurale, l'ambulation précoce et l'apport oral précoce après une chirurgie non urgente pour un cancer colorectal, on pouvait réduire le séjour moyen (Basse, Madsen et Kehlet, 2001; Carli et coll., 2002) et que la mise au point de cheminements cliniques (Melbert et coll., 2002) pour la période suivant cette même chirurgie pouvait réduire, sans danger, le séjour moyen pour les patients.

L'analyse de l'environnement dans notre centre concernant les modèles d'exercice liés aux soins aux patients a montré qu'il était nécessaire d'améliorer la coordination des soins des patients en oncologie chirurgicale et de mettre en place des stratégies de soutien afin de bien préparer les patients et leurs proches à la chirurgie. Deux facteurs clés ont été dégagés pour l'amélioration générale du séjour, soit l'élaboration d'un sommaire des attentes concernant les patients, leur famille et le personnel ainsi que la planification avancée du congé.

En se basant sur les résultats de la recension des écrits et sur les discussions interprofessionnelles, nous avons décidé d'élaborer un document pédagogique initial à l'intention des patients et de leur famille intitulé « Milestones for Colorectal Surgery » [Jalons pour la chirurgie colorectale] (que l'on appellera « Jalons » ci-après). Cet outil servait à coordonner le plan de soins des patients subissant des interventions chirurgicales pour leur cancer colorectal. Dans Jalons, la valeur du séjour moyen indiquait que les patients retournaient à leur domicile en l'espace de six jours (alors que le séjour moyen réel était de huit jours à l'époque), dans l'hypothèse d'une évolution postopératoire sans complications. Un feuillet d'information «Milestones for Colorectal Surgery » destiné aux patients et aux proches a également été conçu. Ce feuillet d'information indiquait clairement les objectifs visés tels que le début ou la fin de thérapies particulières, l'évolution du régime alimentaire des patients et la 
quantité d'activité qui devait être effectuée sur une base journalière. On y présentait des renseignements sur la manière dont les patients et leur famille pouvaient préparer leur domicile en vue du congé et ce, avant même de se rendre à l'hôpital, les objectifs du congé axés sur le patient et les signes et symptômes éventuels qui méritaient d'être signalés au chirurgien après le congé (ainsi que des numéros de téléphone 24/7).

\section{Mise en ouvre de Jalons}

Une fois l'élaboration de Jalons terminée, des séances de formation ont été tenues pour tous les prestataires de soins impliqués dans le processus de préadmission, d'admission et de congé. Le feuillet Jalons a été mis en circulation en janvier 2003. En août 2003, une démarche d'évaluation a été lancée pour ce feuillet sous forme d'enquête auprès des patients et des proches. Cette démarche avait pour but de recueillir les perceptions des patients et des familles relativement aux forces et faiblesses du feuillet Jalons, la fréquence à laquelle les patients l'utilisaient avec leurs proches et les prestataires de soins, et les améliorations qui pourraient y être apportées. Les résultats démontraient que le feuillet Jalons facilitait la discussion entre les patients et leurs proches. En général, les patients et leur famille jugeaient que le matériel pédagogique destiné aux patients leur fournissait des lignes directrices utiles en matière de soins ( $\mathrm{S}$. Moura, B.A. Maier et D. Miller, information personnelle, Février 2006).

La rétroaction des patients et du personnel nous a permis de réviser la version originale de Jalons afin d'y incorporer davantage de renseignements détaillés. Cela a débouché, en juin 2004, sur la préparation de plusieurs feuillets Jalons distincts. Ainsi, des plans de soins particuliers ont été conçus pour 1) hémicolectomie de droite ou de gauche, 2) résection antérieure basse, 3) résection antérieure basse avec iléostomie et 4 ) résection abdomino-périnéale. Cela permettait d'offrir le programme Jalons à l'ensemble des patients subissant une chirurgie colorectale.

Au cours de la période septembre-décembre 2004, le projet a pris un élan important. Henrik Kehlet, l'auteur de plusieurs articles sur le modèle de soins postopératoires en régime accéléré (portant notamment sur les patients en chirurgie colorectale) est venu dans notre établissement et était le conférencier d'honneur à une conférence locale. Le régime accéléré est une approche plurimodale des soins qui met en jeu, le cas échéant, l'analgésie épidurale, la nutrition orale précoce et l'ambulation précoce afin d'améliorer, en toute sûreté, les résultats de la chirurgie et de réduire la durée moyenne du séjour (Kehlet et Wilmore, 2002). Sa présence parmi nous et ses conseils ont donné au personnel impliqué dans le développement du projet un regain d'énergie et d'inspiration. En décembre 2004, une demande de subvention a été adressée au MSSLD de l'Ontario, en vertu de l'initiative Stratégie de réduction des temps d'attente, qui a accordé au projet un financement intégral en mars 2005. Cette subvention a permis d'embaucher une assistante de recherche à temps plein pour la collecte des données quantitatives. La subvention a également fourni le soutien financier nécessaire pour mener une étude qualitative. Une assistante de recherche a été embauchée dont les fonctions consistaient à organiser le groupe de discussion des infirmières, à réaliser les entrevues auprès des patients et à effectuer la transcription de ces dernières.

En mai 2005, le plan de soins Jalons a été mis en œuvre chez tous les patients d'un seul chirurgien admis dans une seule unité dans laquelle œuvrent des infirmières en oncologie chirurgicale spécialisées dans la prestation de soins aux patients subissant une chirurgie pour cancer colorectal. Durant la période allant de juin à août 2005, on demandait aux patients de tenir un journal de bord de leur expérience hospitalière. En juillet, lors de l'arrivée de nouveaux médecins résidents dans notre établissement, nous avons remis au personnel, aux résidents et aux membres des disciplines connexes un document de poche laminé traçant les grandes lignes du plan de soins journaliers des patients (c.-à-d. mini-Jalons). Le mois de septembre 2005 a vu la tenue d'un unique groupe de discussion d'infirmières et le début de la réalisation des entrevues de suivi auprès des patients.
Un rapport documentant les résultats de la facette quantitative du projet Jalons a été envoyé au MSSLD de l'Ontario en décembre tel que stipulé dans l'entente de financement.

\section{But}

Le but de ce projet pilote exploratoire était de décrire l'expérience vécue des patients subissant une chirurgie pour un cancer colorectal ainsi que la perspective des infirmières en oncologie chirurgicale sur les soins dispensés durant une période de quatre mois en 2005, après la mise en œuvre d'un nouveau modèle de soins aux patients fondé sur des données probantes. Ce modèle de soins a été baptisé Jalons.

\section{Méthodologie}

Cette étude qualitative a recueilli des données par l'intermédiaire des journaux de bord des patients, des entrevues auprès des patients et des délibérations d'un groupe de discussion pour infirmières. Une recherche qualitative a été retenue du fait de la nature exploratoire de nos travaux. La théorie ancrée a fourni l'orientation méthodologique nécessaire à l'élaboration du texte de l'entrevue ainsi qu'à la collecte et à l'analyse des données (Hutchinson, 1986).

Entre juin et août 2005, les journaux de bord des patients ont été rassemblés. En septembre 2005, un groupe de discussion a été tenu auprès des infirmières. Enfin, les entrevues de suivi auprès des patients ont eu lieu entre août et octobre 2005 .

\section{Sélection des participants}

\section{Patients}

Les patients admissibles à l'étude étaient ceux qui étaient atteints d'un cancer colorectal et qui subissaient une chirurgie de nature curative non suivie de complications dans le centre de soins tertiaires en cancérologie retenu. L'infirmière en oncologie ambulatoire (BAM) a identifié les patients admissibles dans la clinique d'un unique chirurgien-oncologue. L'infirmière a discuté de l'étude qualitative avec les patients et a transmis, à l'intervieweuse de l'étude $(\mathrm{CF})$, les noms des patients qui se déclaraient prêts à y participer. La coordonnatrice de l'étude communiquait alors avec ces patients et leur expliquait l'étude en se servant d'un modèle normalisé. Les patients étaient priés de tenir un journal de bord dans lequel ils pouvaient commenter leur expérience postopératoire tout au long de leur séjour à l'hôpital et de participer à une entrevue après avoir quitté 1'hôpital. Le consentement à la participation à l'étude qualitative a été obtenu avant la survenue de la chirurgie. Les six patients à qui on a demandé de participer à l'étude ont accepté de le faire.

Les patients étaient exclus s'ils avaient un cancer colorectal avancé localement ou un cancer local récidivant puisqu'on s'attendait à ce qu'ils ne puissent suivre une cure postopératoire permettant de respecter les jalons. Il fallait également que les patients ou leur porteparole consentant aient une maîtrise de l'anglais écrit et oral.

Sur les six patients ayant donné leur consentement, cinq étaient des hommes dont l'âge moyen était de 66 ans (pour une étendue de 46-81 ans). Deux patients ont subi une résection abdomino-périnéale avec colostomie, deux ont subi une résection antérieure basse avec iléostomie, un autre une résection antérieure alors que le sixième a subi une résection antérieure basse avec réparation d'hernies et fermeture de stomie. Chez cinq patients, la douleur était prise en charge à l'aide d'une combinaison d'analgésique épidural thoracique et oral tandis que le dernier patient bénéficiait d'une analgésie contrôlée par le patient et d'analgésiques oraux. Le séjour moyen durait 4,41 jours (pour une étendue de 3-7 jours). Aucun des patients n'a fait l'objet d'une réadmission postopératoire dans les 30 jours. Un patient s'est présenté à l'urgence 15 jours après son congé pour faire enlever ses points de suture.

Toutes les infirmières en oncologie chirurgicale travaillant dans l'unité où les patients subissaient leur chirurgie ont été invitées à participer à un groupe de discussion et ce, par la coordonnatrice d'étude et le chercheur principal. Plusieurs tentatives ont été faites en vue d'organiser une réunion avec l'ensemble du personnel. En fin de 
compte, un seul groupe de discussion a été tenu, auquel six des quarante-six infirmières admissibles ont accepté de participer.

\section{Entrevues auprès des patients}

Les questions ouvertes des entrevues auprès des patients ont été spécifiquement élaborées pour cette étude. Elles ont été mises au point par des médecins et des infirmières qui traitent des patients atteints de cancer colorectal et par des spécialistes en recherche qualitative. Les entrevues téléphoniques se sont déroulées sur une période de trois mois et ont été effectuées par une coordonnatrice d'étude ayant une bonne connaissance de la terminologie des soins de santé $(\mathrm{CF})$. Les entrevues duraient de 30 minutes à deux heures. Les patients ont eu de nombreuses occasions d'exprimer des opinions spontanées et de relater leurs expériences. Les opinions et les expériences ont été explorées en profondeur, et les enjeux communs et particuliers ont été dégagés. Toutes les entrevues ont été enregistrées et transcrites mot à mot ultérieurement.

\section{Journaux de bord des patients}

Les journaux de bord vierges ont été remis aux patients lors de la journée postopératoire 0 . Les journaux de bord posaient plusieurs questions ouvertes aux participants. «De manière générale, commentvous sentez-vous aujourd'hui? », «Qu'est-ce qui va bien pour vous? », «Qu'est-ce qui va mal pour vous? », «Quelles préoccupations avezvous en tête? », « Comment vous sentez-vous aujourd'hui, du point de vue émotionnel? » et enfin une section « Autres commentaires ». Les mêmes questions étaient posées pour chaque jour du séjour à l'hôpital. De plus, le jour où les patients quittaient l'hôpital, on leur demandait de noter toute préoccupation qu'ils avaient à propos de leur congé.

\section{Groupe de discussion des infirmières}

Les questions du groupe de discussion ont également été spécifiquement conçues pour cette étude et ont été élaborées par des médecins et des infirmières traitant des patients atteints de cancer colorectal et par des spécialistes en recherche qualitative. Le groupe de discussion, animé par la coordonnatrice d'étude (CF), a duré environ une heure. Les infirmières en oncologie étaient priées de fournir des renseignements démographiques de base. D'autres questions visaient à cerner les aspects de Jalons qui fonctionnaient bien et ceux qui donnaient de moins bons résultats. On leur demandait également de commenter le rôle qu'elles avaient joué dans le développement et la mise en œuvre de Jalons. Les participantes avaient d'amples opportunités d'exprimer spontanément leurs opinions et leurs expériences. Les opinions et les expériences ont été explorées en profondeur, et les enjeux communs et particuliers ont été cernés. Les échanges du groupe de discussion ont été enregistrés puis transcrits mot à mot.

\section{Analyse des données}

Une fois terminée la transcription des enregistrements des entrevues et de celui du groupe de discussion, les chercheurs (MF, FCW, SM, BAM, JS) et la coordonnatrice d'étude (CF) en ont fait l'analyse. Après avoir effectué une première lecture autonome de chaque transcription et une première prise de notes marginales sur le contenu, les membres de l'équipe se sont rencontrés et ont passé en revue les transcriptions des entrevues, les journaux de bord des patients et les délibérations du groupe de discussion des infirmières. Les membres de l'équipe ont discuté de leurs impressions et se sont mis d'accord sur un schéma de codage du contenu. Une deuxième réunion s'est tenue après qu'une chercheuse (FCW) a organisé des citations représentatives en fonction des champs de contenu. Lors de cette deuxième réunion, l'équipe (MF, FCW, SM, JS, BAM) a réalisé un examen plus approfondi des champs de contenu naissants. Il n'y a guère eu de désaccord au cours de la discussion sur le codage et les quelques champs qui faisaient naître des différends ont été clarifiés en discutant et en explorant la terminologie propre à chacun des codeurs. Cette démarche a débouché sur une réorganisation des champs de contenu (FCW) et sur une nouvelle discussion dans le cadre de la troisième réunion de l'équipe (MF, FCW,
JS, SM, BAM, CF). Pour chacun des champs de contenu codés, les idées principales des patients et du personnel infirmier ont été dégagées et examinées en vue d'obtenir les résultats présentés ci-dessous.

\section{Résultats \\ Patients}

Les six patients invités à tenir un journal de bord postopératoire ont accepté de le faire. L'un des six patients n'a pas pu participer à l'entrevue de suivi en dépit des multiples tentatives par les deux parties de trouver un moment mutuellement convenable. La cassette d'une des entrevues de suivi qui avaient eu lieu a malheureusement été égarée. Tous les patients ont inscrit des commentaires dans leur journal de bord pour chacune des journées de leur hospitalisation, y compris celle de leur chirurgie. Un patient a fourni des notes détaillées sur son humeur et sur ses attentes entre son réveil le jour de son opération et les instants précédant son entrée dans la salle d'opération. Ce patient a recommencé à ajouter des renseignements dans son journal de bord dans la salle de réveil. Dans l'ensemble, les inscriptions gagnaient en longueur et en détail avec le passage du temps. Les données issues des patients ont permis de dégager six champs de contenu.

\section{Champ de contenu 1 : Recherche d'information - Différents besoins pré- et postopératoires}

Les patients ont signalé que leurs besoins en matière d'information avaient été satisfaits avant leur opération du fait de leurs propres recherches et du soutien informationnel des cliniques (chirurgie, évaluation préliminaire). Par contre, leurs besoins d'information postopératoires n'avaient pas été satisfaits, notamment en ce qui concerne les plans de congé. Les patients indiquaient qu'ils avaient besoin de renseignements particuliers se rapportant directement à leur situation individuelle et que ces renseignements ne leur étaient pas fournis.

\section{Champ de contenu 2 : Satisfaction des attentes} relatives à la maîtrise de la douleur postopératoire

Les patients se déclaraient surpris du peu de douleur qu'ils avaient ressenti après l'opération. Face à une chirurgie de cette ampleur, ils s'attendaient à éprouver de la douleur et à avoir du mal à sortir de leur lit.

\section{Champ de contenu 3 :}

\section{Reconnaissance à l'égard des professionnels de la santé}

Les patients décrivaient l'importante gratitude qu'ils ressentaient envers le personnel de la santé (chirurgiens et infirmières). Ce sentiment de reconnaissance naissait de la réalisation qu'ils étaient atteints du cancer et qu'ils venaient de subir une opération lourde.

\section{Champ de contenu 4 : Prêts à rentrer au domicile}

Les patients manifestaient de l'enthousiasme à l'idée de rentrer chez eux et de revoir leurs proches au moment du congé (cinquième ou sixième jour après l'opération). Ils rapportaient en outre qu'il leur avait été utile de pouvoir comparer leur propre progrès postopératoire aux indications de Jalons et que cela leur permettait d'anticiper le moment où ils recevraient leur congé.

\section{Champ de contenu 5 : Obstacles aux soins-facteurs liés au système}

Quoique les patients étaient heureux des résultats de la chirurgie et des soins infirmiers qui leur avaient été dispensés, ils faisaient remarquer que certains facteurs associés au système avaient rendu leurs soins plus stressants. Ils faisaient état, entre autres, de l'attente liée à l'arrivée des fournitures pour les soins à domicile et à la préparation des prescriptions au départ et du manque de clarté entourant les rendezvous de suivi à la clinique de cancérologie après la sortie de l'hôpital.

\section{Champ de contenu 6 : Conseils à l'équipe}

Les patients ont donné des conseils à l'équipe sur l'amélioration possible des soins prodigués. Leurs suggestions comprenaient la mise en place de systèmes de suppléance lorsque les chirurgiens prennent des vacances, la clarification de l'information postopératoire et l'accroissement du soutien dispensé dans le cadre des soins à domicile. 


\section{Infirmières}

Six des 46 infirmières qui œuvraient dans une même unité de chirurgie ont participé au groupe de discussion. Huit champs de contenu ont été cernés grâce à l'examen de la transcription de la séance de discussion.

\section{Champ de contenu 1 : Aspects positifs du projet Jalons}

Les infirmières ont décrit qu'elles aimaient avoir une idée nette de l'évolution postopératoire prévue pour un patient donné et que ce savoir leur permettait de jouer un rôle important au sein de l'équipe des soins aux patients. Elles ont ajouté qu'elles pensaient que cette information était également bénéfique pour les patients puisqu'ils comprenaient ce à quoi ils pouvaient s'attendre tout au long de leur rétablissement postopératoire. Les infirmières croyaient que cela motivait les patients à atteindre leurs objectifs quotidiens en matière de rétablissement.

\section{Champ de contenu 2 : Aspects négatifs du projet Jalons}

Les infirmières ont indiqué que le projet Jalons pourrait avoir une incidence négative. Elles ont déclaré qu'il pourrait engendrer de l'anxiété chez les patients si ceux-ci n'atteignaient pas les objectifs quotidiens prescrits. Les infirmières éprouvent également de l'anxiété s'il leur est impossible d'aider les patients à atteindre ces objectifs.

\section{Champ de contenu 3 : Aliénation}

Les infirmières jugeaient qu'elles n'avaient été impliquées ni dans le processus de planification ni dans la mise en œuvre du projet Jalons dans leur service. Selon elles, une implication dans ces étapes préliminaires les aurait aidées à comprendre les buts du projet et leur rôle au niveau de leur atteinte. Ainsi, elles auraient eu davantage l'impression d'être des partenaires à part entière du projet.

\section{Champ de contenu 4 : Elles souhaitaient apporter leur contribution}

Les infirmières participantes estimaient que le projet aurait pu tirer parti de leurs idées et suggestions. Elles pensaient posséder une expertise dans le domaine des soins aux patients atteints d'un cancer colorectal, laquelle aurait pu être incorporée avantageusement dans le document.

\section{Champ de contenu 5 : Points de vue contradictoires sur l'incidence du projet Jalons sur la pratique}

Des points de vue contradictoires sont apparus au sein du groupe de discussion en ce qui a trait à l'incidence du projet Jalons sur les pratiques de travail des infirmières. Certaines infirmières déclaraient qu'il n'avait ni modifié leur pratique ni eu de répercussions sur leur charge de travail; en revanche, d'autres décrivaient le surcrô̂t d'attention qu'obtenaient les patients relevant du projet Jalons (p. ex. ils veillaient à recevoir une aide ambulatoire afin d'atteindre les jalons prescrits) et que cela augmentait leur charge de travail.

\section{Champ de contenu 6 : Conseils aux chercheurs}

Les infirmières mettaient de l'avant diverses suggestions pratiques qui permettraient d'accroître le nombre de patients atteignant les buts associés aux Jalons quotidiens et d'éveiller l'enthousiasme et de favoriser la participation du personnel infirmier au projet. Selon quelques-uns de leurs conseils, elles souhaitaient qu'on leur fournisse les preuves issues des écrits scientifiques ayant orienté le changement de pratique, plutôt que de leur présenter seulement les résultats de la démarche. Les infirmières ont également suggéré qu'il fallait qu'elles puissent participer plus activement aux étapes de planification et pas uniquement à la composante de mise en œuvre.

\section{Champ de contenu 7 : Obstacles au projet Jalons}

Les infirmières ont mentionné plusieurs obstacles à l'atteinte des buts précisés dans Jalons dont la difficulté de prodiguer des soins équitables aux patients qui leur sont assignés, particulièrement lorsque certains d'entre eux sont gravement malades et d'autres prennent bien plus de temps pour arriver dans l'unité après la chirurgie.
Champ de contenu 8 : Intérêt pour les soins

et la recherche fondés sur des résultats probants

Les infirmières ont montré qu'elles voulaient avoir accès à la documentation sur laquelle se basait le changement de pratique et être impliquées dans la recherche sur les soins aux patients. Elles exprimaient le vif désir d'améliorer la pratique.

\section{Discussion}

Dans cette étude, nous avons décrit les expériences des patients tandis qu'ils subissaient une opération pour leur cancer colorectal ainsi que les expériences des infirmières qui les soignaient dans le cadre du projet Jalons.

\section{Données relatives aux patients}

L'un des buts premiers de cette étude était de recueillir la rétroaction des patients à l'égard de Jalons. Il est intéressant de noter que les patients n'ont pas fait directement référence au feuillet Jalons en tant que tels ni dans leurs journaux de bord ni au cours de l'entrevue, à moins d'y avoir été invités par l'intervieweuse. Il est possible que ceci s'explique en partie par le fait qu'il s'agissait, pour les patients, de leur première chirurgie colorectale et que l'expérience était entièrement nouvelle à leurs yeux. De plus, il était question des jalons dans toute l'information qu'ils recevaient de notre organisme et ils ignoraient donc qu'un autre type de programme pouvait être offert. Ils ont trouvé que le document était utile puisqu'il leur permettait de mesurer leurs progrès. Même en restant moins longtemps à l'hôpital que leurs homologues des cohortes précédentes, ils disaient avoir hâte de pouvoir rentrer chez eux dans des délais plus brefs que ceux mentionnés dans Jalons. Cela contrastait avec la supposition émise par les chercheurs selon laquelle les patients auraient pu se sentir brusqués de devoir quitter l'hôpital, étant donné la plus grande brièveté du séjour moyen indiqué dans Jalons.

Les patients se déclaraient très satisfaits de la quantité d'information dont ils disposaient avant la chirurgie. Autre fait surprenant, pour les chercheurs de l'étude du moins, était la quantité d'information que les patients et leurs proches avaient rassemblée auprès de sites Internet avant la survenue de l'opération. À l'opposé de la satisfaction de leurs besoins préopératoires, les patients exprimaient le besoin de recevoir davantage d'information sur les soins postopératoires et que celle-ci soit à la fois brève et individualisée. Un récent exposé de synthèse sur les besoins et les ressources en matière d'information chez les patients en cancérologie (Rutten, Arora, Bakos, Aziz et Rowland, 2005) faisait état de résultats semblables, en précisant que les besoins d'information des patients changent au fil du temps.

Les écrits de date récente indiquent que le risque d'événements indésirables est plus élevé post-congé que durant l'hospitalisation et qu'il existe souvent une lacune au niveau de la transition de l'hôpital au domicile (Elwyn, Forster et Freeman, 2005). Cette lacune est associée à un manque de planification de congé qui soit claire, concise, axée sur le patient et dont la démarche implique le patient et sa famille (Elwyn et coll., 2005; Grimmer, Moss, Falco et Kindness, 2006). Dans notre étude, les obstacles aux soins rapportés par les patients concernaient principalement le processus de congé, notamment l'attente des fournitures et des prescriptions des médecins. Une récente étude (Fung et Lai, 2006) signale que la frustration ressentie envers le processus de congé ne se limite pas aux patients, les prestataires de soins participant à la planification des congés trouvent, eux aussi, que ce processus est mal coordonnée et source de frustration. Des auteurs ont récemment tenté de déterminer les besoins des patients qui devraient être satisfaits dans le cadre d'un processus de congé réussi (Grimmer et coll., 2006). Un examen détaillé de notre processus de planification des congés devrait être une des priorités futures de cette équipe.

Malgré ces inquiétudes, les patients étaient très reconnaissants envers les professionnels de la santé. Un autre aspect positif de la rétroaction des patients était le peu de douleur qu'ils rapportaient, surtout qu'ils avaient tous subi une chirurgie abdominale lourde. Il est vrai que l'hôpital est doté d'un service de prise en charge de la douleur aiguë. 


\section{Personnel infirmier}

Le personnel infirmier en oncologie chirurgicale interviewé voyait dans Jalons un document utile, à la fois pour lui-même et pour les autres membres de l'équipe de soins, mais surtout pour les patients. Cependant, les infirmières ont signalé un sentiment d'aliénation et de manque d'implication au niveau du processus de planification et ont exprimé leur désir d'y apporter leur contribution. Ces attitudes ont surpris les chercheurs de l'étude. Ainsi, au moins une infirmière participant directement aux soins aux patients assistait à chacune des réunions tenues avant la mise en œuvre de Jalons et nous faisions consciemment l'effort d'inviter les commentaires et suggestions de tous les intervenants assistant à ces réunions. De plus, l'initiative Jalons figurait à l'ordre du jour des réunions mensuelles du personnel. Même aujourd'hui, nous ignorons comment l'information a été transmise à l'ensemble du personnel infirmier, voire si elle l'a été du tout. Un mécanisme de rétroaction bidirectionnelle n'a pas été mis en œuvre. Les données de cette étude pilote suggèrent une rupture manifeste des communications entre les infirmières qui prodiguaient les soins aux patients et aux proches et les chercheurs de l'étude, et ce domaine pourra faire l'objet d'une amélioration significative. Toutefois, il était difficile de trouver des infirmières de chevet qui pouvaient donner de leur temps au projet à son stade initial. Il convient de remarquer, également, qu'en dépit de tentatives répétées de recrutement d'infirmières pour le groupe de discussion (primes d'encouragement financières et alimentaires), sur une période de trois mois, nous n'avons pu recruter que 6 des 46 membres du personnel. L'envoi d'un questionnaire à chaque membre du personnel en vue de cerner les dates et heures qui conviendraient le mieux pour la participation au groupe de discussion est resté sans réponse. Cela illustre les défis qui doivent être surmontés quand on essaie de déterminer la manière dont on peut impliquer, dans des activités autres que les soins directs aux patients, le personnel infirmier de première ligne œuvrant dans une unité de chirurgie très fréquentée. Il est évident que cette question mérite un examen plus approfondi. Pour conclure sur ce sujet, bien que les infirmières de première ligne aient fort peu participé aux étapes initiales, elles ont proposé un éventail de suggestions pratiques aux retombées positives pour les soins aux patients peu après la mise en œuvre de Jalons.

Les infirmières de chevet ont également indiqué qu'elles auraient aimé avoir à leur disposition une partie de la documentation scientifique afin qu'elles puissent mieux apprécier la raison d'être de ce changement de pratique. Les responsables des futures recherches touchant les soins aux patients devraient s'efforcer de trouver des moyens de solliciter les commentaires et suggestions des infirmières durant l'étape de planification plutôt que de les obtenir durant celle de la mise en œuvre. Il est fort possible que nous n'aurions reçu aucune rétroaction formelle des infirmières de chevet si nous n'avions pas mis sur pied le groupe de discussion, du fait de notre incapacité à engager la participation du personnel infirmier par le biais d'autres mécanismes de rétroaction (courriel, conversations de couloir, diffusion des données dans l'unité sous forme d'affiches). Cette équipe de recherche est prête à reconnaître que ses efforts visant à recueillir les commentaires et suggestions du personnel infirmier n'ont guère abouti et qu'il convient d'étudier et d'utiliser d'autres méthodes.

Une limite manifeste de cette étude est la taille réduite de l'échantillon de patients et du faible nombre d'infirmières recrutées. Les membres de l'équipe avaient espéré pouvoir recruter davantage de patients, mais plusieurs facteurs relatifs à l'établissement ont fait du recrutement une tâche plus ardue que prévue. Ainsi, le recrutement se déroulait avant tout pendant l'été. Divers membres de l'équipe ont pris leurs vacances en cette saison et ont été absents pendant de longues périodes. De plus, une seule infirmière était disponible pour recruter les patients. Les aléas du fonctionnement d'un grand hôpital d'enseignement ont également compliqué le travail de recrutement de patients. Ainsi, les résidents en chirurgie de notre ville changent d'hôpital en janvier et en juillet chaque année. Avant de pouvoir se consacrer au recrutement de patients, notre équipe devait donc s'assurer que les nouveaux résidents de chirurgie étaient au courant de Jalons et qu'ils allaient utiliser le programme. De toute manière, la taille réduite de l'échantillon n'a pas empêché l'équipe de dégager des aperçus très pointus des expériences vécues des patients et des infirmières dans ce contexte chirurgical particulier.

\section{Conclusion}

En dépit de sa moindre envergure, cette étude pilote exploratoire a fourni des aperçus d'une grande perspicacité de l'expérience vécue des patients subissant un traitement chirurgical de leur cancer colorectal ainsi que des aperçus pointus de l'expérience des infirmières en oncologie chirurgicale qui leur prodiguent des soins après l'institution d'un nouveau protocole de soins aux patients. Dans l'ensemble, la mise en œuvre de Jalons constitue une réussite. Cette étude pilote a démontré qu'il fallait explorer notre processus de planification des congés et les méthodes permettant d'impliquer activement les infirmières de chevet dans les étapes de planification des projets à venir. Une autre initiative que le groupe sur le cancer colorectal pourrait entreprendre est l'évaluation de certains des sites Internet qui fournissent des renseignements sur la chirurgie colorectale et la création d'une liste de sites qui présentent des informations claires, concises et surtout valides et fiables.

\section{References}

Basse, L., Hjort Jakobsen, D., Billesbolle, P., Werner, M., \& Kehlet, H. (2000). A clinical pathway to accelerate recovery after colonic resection. Ann Surg, 232(1), 51-57.

Basse, L., Madsen, J.L., \& Kehlet, H. (2001). Normal gastrointestinal transit after colonic resection using epidural analgesia, enforced oral nutrition and laxative. Br J Surg, 88(11), 1498-1500.

Basse, L., Raskov, H.H., Hjort Jakobsen, D., Sonne, E., Billesbolle, P., Hendel, H.W., et al. (2002). Accelerated postoperative recovery programme after colonic resection improves physical performance, pulmonary function and body composition. $\mathbf{B r} \mathbf{J}$ Surg, 89(4), 446-453.

Carli, F., Mayo, N., Klubien, K., Schricker, T., Trudel, J., \& Belliveau, P. (2002). Epidural analgesia enhances functional exercise capacity and health-related quality of life after colonic surgery: Results of a randomized trial. Anesthesiology, 97(3), 540-549.

Elwyn, G., Forster, A., \& Freeman, G. (2005). Mind the gap: The risk of adverse events and errors during patient discharge [Electronic Version]. BMJ Publishing Group, 6. Retrieved July 27, 2005, from www.saferhealthcare.org.uk
Fung, I., \& Lai, D. (2006, March). Identifying barriers for oncology patients in accessing rehabilitation: A qualitative study. University of Toronto Medical Journal, 78-82.

Grimmer, K., Moss, J., Falco, J., \& Kindness, H. (2006). Incorporating patient and carer concerns in discharge plans: The development of a practical patient-centered checklist [Electronic Version]. The Internet Journal of Allied Health Sciences and Practice, 4, 1-8.

Hutchinson, S. (1986). Nursing research-A qualitative perspective. Norwalk.

Kehlet, H., \& Wilmore, D.W. (2002). Multimodal strategies to improve surgical outcome. Am J Surg, 183(6), 630-641.

Melbert, R.B., Kimmins, M.H., Isler, J.T., Billingham, R.P., Lawton, D., Salvadalena, G., et al. (2002). Use of a critical pathway for colon resections. J Gastrointest Surg, 6(5), 745-752.

Rutten, L.J., Arora, N.K., Bakos, A.D., Aziz, N., \& Rowland, J. (2005). Information needs and sources of information among cancer patients: A systematic review of research (1980-2003). Patient Educ Couns, 57(3), 250-261. 